\title{
Is There a Demand for Income Tax Progressivity?
}

\author{
Jean Hindriks* \\ Queen Mary and Westfield College \\ University of London
}

8 June 2000

\begin{abstract}
Recently Marhuenda and Ortuno-Ortin (1995) have provided a popular support for progressivity theorem that says that a marginal progressive tax always defeats a marginal regressive tax as long as individuals vote for the tax scheme minimizing their tax liabilities and the median income is less than the mean income. In this paper we provide, under similar circumstances, a popular support for regressivity theorem according to which more marginal regressivity (or less marginal progressivity) can always defeat any existing tax scheme. This move towards more regressivity (or less progressivity) is supported by the extremes of the income distribution. Combining this result with Marhuenda and Ortuno-Ortin' result implies that vote cycling is inevitable and that the demand for progressivity cannot be established in the standard Downsian framework with self-interested voters.
\end{abstract}

Keywords:Voting, Redistribution, Income tax progresivity JEL classification: D72

* Economics department, QMW,University of London, Mile End Road, London E1 4NS, England. Tel. (44)-020-78827807, Email:j.hindriks@qmw.ac.uk. 


\section{Introduction}

Optimal taxation cannot explain the apparent demand for progressivity in developped countries. Indeed, tax policies derived from some social welfare optimization can be either (marginal) progressive or regressive (see Myles, 2000). However, if we agree that voters care about the fairness of taxation rather than maximizing social welfare, then Young (1990) has shown that equal sacrifice implies progressiveness. But such arguments typically fail if voters are self-interested. Adopting the Downsian framework, with twoparty without ideology competing to win the election and self-interested voters, both Snyder and Kramer (1988) and Cukierman and Meltzer (1991) obtain the existence of a Condorcet winner involving progressive taxation, but only under rather strong and mainly unjustifiable conditions. ${ }^{1}$

The main reason why the Downsian approach has failed to explain the observed democratic demand for progressivity is that voting over non-linear income tax policies requires a policy space that is at least two-dimensional. Therefore we cannot put the alternatives in a transitive order and we do not generally expect to get Condorcet winner. It follows that any tax policy could be defeated by at least one other policy. Recently, Marhuenda and Ortuno-Ortin (1995) have suggested one interesting way out. They consider simple voting over two tax schemes: an arbitrary status quo and an amendment. This formulation avoids the difficulties of multi-dimensional voting and still retains the essential aspects of majority voting over tax policies. Moreover this formulation enables them to obtain interesting insights on the democratic demand for progressivity. Indeed they obtain the remarkable result that any marginal progressive tax wins over any regressive one provided that the median is less than the mean income. This popular support for progressivity theorem is obtained with self-interested voters who vote for the tax policy that taxes them less. ${ }^{2}$

The purpose of this paper is to present under similar circumstances a popular support for regressivity theorem according to which any tax scheme can be defeated by a less marginal progressive (or more marginal regressive) tax scheme supported by a majority of the extremes. Combining this regressiv-

\footnotetext{
${ }^{1}$ For instance Snyder and Kramer (1988) reduce the policy space to tax schemes that are preferred by some voters.

${ }^{2}$ The result has been generalized by Mitra et al (1998) to more sophisticated voters who also care about their relative position in the income distribution.
} 
ity theorem with the progressivity theorem establishes the inevitable voting cycle Marhuenda and Ortuno-Ortin were trying to escape. ${ }^{3}$

\section{The Model}

We consider a one good economy (consumption) populated by a large number of individuals who differ only in their income levels. Each individual is characterized by her fixed income level, $y \in \Omega=[0, Y]$. (Thus the highest income level in the economy is $Y$.) The distribution of income in the population is described by a strictly increasing distribution function $F$ on $[0, Y]$, so that $F(y)$ is the fraction of the population with pre-tax income less or equal to $y$. The mean income is $\bar{y}=\int_{\Omega} y d F(y)$ and the median income is $y_{m}=F^{-1}(1 / 2)$. We assume throughout that $y_{m} \leq \bar{y}$. The tax liability of an individual with pre-tax income $y$ is $t(y)$ where $t: \Omega \rightarrow R$ is a continuous tax function satisfying the following feasibility conditions:

$$
\begin{gathered}
t(y) \leq y \quad \text { for all } \quad y \in[0, Y] \\
\int_{\Omega} t(y) d F(y)=0
\end{gathered}
$$

Condition (1) says that tax liabilities cannot exceed taxable income. Condition (2) is merely a budget balance condition with zero revenue requirement (i.e., purely redistributive taxation).

Voting over both progressive and regressive tax schemes requires a policy space that is at least two-dimensional. In the following we shall consider quadratic income taxes of the form:

$$
t(y)=-c+b y+a y^{2},
$$

where $c \geq 0$ is the uniform lump-sum transfer, $b$ is the linear tax parameter (with $0 \leq b \leq 1)$, and $a>0(<0)$ is the progressivity (regressivity) tax parameter. Let $X$ be the set of quadratic tax functions that satisfy the feasibility conditions (1) and (2). Using the budget balance condition (2), we can express $c$ as a function of $a$ and $b$ :

$$
\begin{aligned}
c & =b \bar{y}+a \bar{y}_{2} \\
& =b \bar{y}+a\left(\bar{y}^{2}+\sigma^{2}\right) .
\end{aligned}
$$

\footnotetext{
${ }^{3}$ We should note that Marhuenda and Ortuno-Ortin make in fact allusion to this possibility in their conclusion.
} 
where $\bar{y}_{2}=\int_{\Omega} y^{2} d F(y)$ and $\sigma^{2}=\bar{y}_{2}-\bar{y}^{2}$ is the variance of the income distribution. So, tax policies are two-dimensional, $(a, b)$.

Democratic countries typically rely on political parties to select a small subset of the possible fiscal policies, and then only this small selected set of fiscal policies will be considered by the voters in the general election. Let us consider a simple model of how political parties select fiscal policies. For simplicity, we also assume here that there are only two political parties without ideology who simply wish to win the election by selecting a fiscal policy preferred by a majority to the one selected by the other party. They must choose their policy simultaneously and independently. This is the standard Downsian majority voting game. Let $P$ denote the majority preference relation. Assuming an odd number of voters, the majority preference $P$ is a binary relation satisfying the asymmetry and completeness properties of a tournament. Supposing that voters are simply voting for the tax scheme that taxes them less, the majority preference relation over any tax pair $\left(t_{1}, t_{2}\right) \in X^{2}$ is given by,

$$
\begin{array}{ccc}
t_{1} P t_{2}: & n\left(t_{1}, t_{2}\right)>n\left(t_{2}, t_{1}\right) \\
t_{2} P t_{1}: & n\left(t_{1}, t_{2}\right)<n\left(t_{2}, t_{1}\right),
\end{array}
$$

where $n\left(t_{1}, t_{2}\right)=\#\left\{y \in[0, Y]: t_{1}(y) \leq t_{2}(y)\right\}$ is the number of voters who (weakly) prefer $t_{1}$ to $t_{2}$, and $n\left(t_{2}, t_{1}\right)=\#\left\{y \in[0, Y]: t_{1}(y)>t_{2}(y)\right\}$ is the number of voters who prefer $t_{2}$ to $t_{1}$.

If the two parties choose different fiscal policies, then the one which selects the policy preferred by a majority of voters wins the election. Otherwise, each party wins with probability $1 / 2$. Considering that each party is only interested to win and can choose among the same set of admissible policies, we have a symmetric two-player zero-sum game $G=(X, X, U)$ where:

$$
U\left(t_{1}, t_{2}\right)=\left\{\begin{array}{rlr}
1 & : & t_{1} P t_{2} \\
-1 & : & t_{2} P t_{1} \\
0 & : & t_{1}=t_{2}
\end{array}\right.
$$

This game, called the majority game, has a unique Nash equilibrium in pure strategies if and only if there exists a Condorcet winner, that is a policy $t^{*} \in X$ such that $t^{*} P t$ for all $t \in X \backslash\left\{t^{*}\right\}$. But because the set of policy alternatives is bi-dimensional, we cannot put the alternatives in a transitive order and we do not generally expect to get a Condorcet winner. This is 
exactly what we are going to prove. For any (positively skewed) income distribution, there is no Condorcet winner and any policy could be defeated by at least one other policy (either more or less progressive). Note that this is for a reduced policy space involving only quadratic tax functions, and thus, a fortiori it must be true for more general continuous tax schemes. It follows that the game cannot have any equilibria in pure strategies, because each party could win the election if it knew which policy would be chosen by the other party.

We establish the inevitable voting cycle by means of two propositions. Proposition 1 states that any feasible quadratic tax scheme $t_{2} \in X$ can be defeated under a majority coalition of the extremes by a less progressive (or more regressive) feasible tax scheme $t_{1} \in X$. Then Proposition 2 states that any feasible quadratic tax scheme $t_{2} \in X$ can be defeated under a majority coalition of the poor and middle class by a more progressive (or less regressive) feasible tax scheme $t_{1} \in X$. Of course Proposition 2 is a simple restatement to quadratic tax schemes of Marhuenda and Ortuno-Ortin' popular support for progressivity theorem.

\section{Inevitable voting cycle}

To prove Proposition 1 we need the following Lemma which makes precise the necessary and sufficient conditions for any tax scheme to be defeated by a less progressive one.

Lemma: Suppose income is fixed and distributed according to $F(y)$ in $[0, Y]$, with $y_{m} \leq \bar{y}$. Then for any tax scheme $t_{2}=-c_{2}+b_{2} y+a_{2} y^{2}$ there exists a less progressive tax scheme $t_{1}=-c_{1}+b_{1} y+a_{1} y^{2}$ with $a_{1}<a_{2}$, $b_{1}>b_{2}$ and $c_{1}>c_{2}$ such that $t_{1} P t_{2}$ if and only if there exists a scalar $\alpha>0$ such that:

$$
\begin{aligned}
& F\left(\alpha \bar{y}+\sqrt{(\alpha-1)^{2} \bar{y}^{2}+\sigma^{2}}\right)-F\left(\alpha \bar{y}-\sqrt{(\alpha-1)^{2} \bar{y}^{2}+\sigma^{2}}\right)<1 / 2 \\
& \quad \alpha \bar{y} \pm \sqrt{(\alpha-1)^{2} \bar{y}^{2}+\sigma^{2}} \quad \in[0, Y] \\
& \quad \alpha>\frac{1}{2}+\frac{\sigma^{2}}{2 \bar{y}^{2}}
\end{aligned}
$$

Proof: Consider the two tax schedules: $t_{1}=-c_{1}+b_{1} y+a_{1} y^{2}$ and $t_{2}=-c_{2}+b_{2} y+a_{2} y^{2}$ and let $T=t_{1}-t_{2}=-c+b y+a y^{2}$ with $a=a_{1}-a_{2}<0$, $b=b_{1}-b_{2}>0$, and $c=c_{1}-c_{2}>0$. From the budget balance constraint, we have $c=b \bar{y}+a \bar{y}_{2}$. Clearly, $T$ is concave with a negative intercept and two real roots: 


$$
\left(y_{1}, y_{2}\right)=y^{*} \pm \frac{\sqrt{b^{2}+4 a c}}{2 a} .
$$

with $y^{*}=-\frac{b}{2 a}$ and $b^{2}+4 a c>0$. Therefore, all those with income $y \in\left[y_{1}, y_{2}\right]$ are paying more taxes under $t_{1}$ and all those outside this interval pay less taxes under $t_{1}$. Now fix $y^{*}=\alpha \bar{y}$ with $\alpha>0$; that is the interval $\left[y_{1}, y_{2}\right]$ is centered around $\alpha \bar{y}$. It follows that $b=-2 a \alpha \bar{y}$ and thus we get $^{4}$,

$$
\left(y_{1}, y_{2}\right)=\alpha \bar{y} \pm \sqrt{(\alpha-1)^{2} \bar{y}^{2}+\sigma^{2}}
$$

It follows that $t_{1} P t_{2}$ is equivalent to

$$
F\left(\alpha \bar{y}+\sqrt{(\alpha-1)^{2} \bar{y}^{2}+\sigma^{2}}\right)-F\left(\alpha \bar{y}-\sqrt{(\alpha-1)^{2} \bar{y}^{2}+\sigma^{2}}\right)<1 / 2
$$

which is condition $(\mathrm{A})$ of the proposition. Furthermore $\left(y_{1}, y_{2}\right) \in[0, Y]$ only if Condition (B) of the proposition is satisfied. Finally using $b=-2 a \alpha \bar{y}$, the requirement $c>0$ is equivalent to

$$
c=-2 a \alpha \bar{y}^{2}+a\left(\bar{y}^{2}+\sigma^{2}\right)>0
$$

or (given $a<0)$

$$
\alpha>\frac{1}{2}+\frac{\sigma^{2}}{2 \bar{y}^{2}}
$$

which is Condition $(\mathrm{C})$ in the proposition. QED.

Using this Lemma we can now prove the following proposition,

Proposition 1: Suppose income is fixed and distributed according to $F(y)$ in $[0, Y]$, with $y_{m}<\bar{y}$ and $Y$ large enough. Then for any tax scheme $t_{2}=-c_{2}+b_{2} y+a_{2} y^{2}$ there exists a less progressive tax scheme $t_{1}=$ $-c_{1}+b_{1} y+a_{1} y^{2}$ with $a_{1}<a_{2}, b_{1}>b_{2}$ and $c_{1}>c_{2}$ such that $t_{1} P t_{2}$.

Proof: Let us fix $\alpha$ so that the lower bound of the interval $\left[y_{1}, y_{2}\right]$ coincides with $y_{m}$ (with $y_{m}<\bar{y}$ ), and then conditon (A) is automatically satisfied since necessarily more than half of voters would fall below the interval. Formally, set $\alpha>0$ such that

$$
y_{m}=\alpha \bar{y}-\sqrt{(\alpha-1)^{2} \bar{y}^{2}+\sigma^{2}}
$$

\footnotetext{
${ }^{4} \mathrm{Using} b=-2 a \alpha \bar{y}$ and $c=b \bar{y}+a\left(\bar{y}^{2}+\sigma^{2}\right)$ we have $b^{2}+4 a c=4 a^{2} \alpha^{2} \bar{y}^{2}-8 a^{2} \alpha \bar{y}^{2}+$ $4 a^{2}\left(\bar{y}^{2}+\sigma^{2}\right)=4 a^{2} \bar{y}^{2}\left(\alpha^{2}-2 \alpha+1\right)+4 a^{2} \sigma^{2}=4 a^{2}\left[(\alpha-1)^{2} \bar{y}^{2}+\sigma^{2}\right]>0$ for all $\alpha>0$
} 
or equivalently

$$
(\alpha-1) \bar{y}+\left(\bar{y}-y_{m}\right)=\sqrt{(\alpha-1)^{2} \bar{y}^{2}+\sigma^{2}}
$$

which is possible since by assumption $y_{m}<\bar{y}$. This reduces to

$$
\alpha=1+\frac{\sigma}{2 \bar{y}}\left(\frac{1-s^{2}}{s}\right) .
$$

where $s=\left(\bar{y}-y_{m}\right) / \sigma>0$ denotes the measure of skewness (with $0<s \leq 1$ ) and we have used the fact that $\bar{y}-y_{m}=s \sigma$. It can be checked that Condition (C) is automatically satisfied for this value of $\alpha$. Lastly Condition (B) is met for any $s>0$ provided that $Y$ is large enough. QED

Notice that this result holds true regardless of the form of the probability distribution function $F(y)$, provided that the median is less than the mean income. ${ }^{5}$ The result is not trivial because the curvature of the tax function affects the intercept, and the distribution of income influences the length of the interval $\left[y_{1}, y_{2}\right]$ : the lower the probability mass around $\bar{y}$ (always contained in the interval) the longer the interval.

The next proposition, adapted from Marhuenda and Ortuno-Ortin (1995), establishes the inevitable voting cycle.

Proposition 2: Suppose income is fixed and distributed according to $F(y)$ in $[0, Y]$, with $y_{m} \leq \bar{y}$. Then for any tax scheme $t_{2}=-c_{2}+b_{2} y+a_{2} y^{2}$ there exists a more progressive tax scheme $t_{1}=-c_{1}+b_{1} y+a_{1} y^{2}$ with $a_{1}>a_{2}$, $b_{1}<(>) b_{2}$ and $c_{1}>c_{2}$ such that $t_{1} P t_{2}$.

Proof: Consider the two tax schedules $t_{1}=-c_{1}+b_{1} y+a_{1} y^{2}$ and $t_{2}=$ $-c_{2}+b_{2} y+a_{2} y^{2}$ and let $T=t_{1}-t_{2}=-c+b y+a y^{2}$ with $a=a_{1}-a_{2}>0$, $b=b_{1}-b_{2}<(>) 0$, and $c=c_{1}-c_{2}>0$. From the balanced budget constraint, we have $c=b \bar{y}+a \bar{y}_{2}$. Clearly, $T$ is convex with a negative intercept. Since $T$ is strictly convex, using Jensen's inequality we get,

$$
T(\bar{y})=T\left(\int_{\Omega} y d F(y)\right)<\int_{\Omega} T(y) d F(y)=0
$$

So, $T(\bar{y})<0$ and since $T$ is strictly convex, $T$ must be strictly increasing

\footnotetext{
${ }^{5}$ In fact, it can be shown that the result fails for symmetric distributions like the uniform or the triangular ones. Proof of this statement is available upon request.
} 
and therefore $T(y)<0$ for all $y \in[0, \bar{y}]$, that is all those with income below the mean income pay less taxes under $t_{1}$ than under $t_{2}$. Since $y_{m} \leq \bar{y}$ more than half the voters would prefer $t_{1}$ to $t_{2}$ and the result follows. QED

\section{Conclusion}

In this paper we have supplemented the popular support for progressivity theorem of Marhuenda and Ortuno-Ortin (1995) with a novel popular support for regressivity theorem to establish the inevitable voting cycle over tax policies. There are three possible solutions to this difficulty. Firstly we can abandon the Downsian assumption that parties are only interested to win election, but the problem is that the Condorcet winner may not be selected if it exists. Secondly, we can keep the Downsian approach but adopt alternative solution concepts that are Condorcet consistent in the sense that they will pick the Condorcet winner if any as the unique outcome of the game (examples of such solution concepts are the uncovered set,the minimal covering set, the Bipartisan set and the Banks set) ${ }^{6}$. Thirdly we can adopt alternative rules for the voting game. For instance we can consider issue-by-issue voting or sequential voting. The first approach has been carried out forcefully by Roemer (1999). The two other approaches have been investigated among other by De Donder and Hindriks (2000).

Acknowledgements. This paper was initiated while I was visiting Institut d'Economie Industrielle and GREMAQ, Toulouse (July 1999). Financial support from the University of Toulouse is gratefully acknowledged. I would like to thank Philippe De Donder and Gareth Myles for helpful comments and suggestions. I also owe special thanks to Paul Belleflamme for pointing out an error in the previous version of the paper and making suggestions that lead to a significant improvement of the paper.

\section{References}

[1] Cukierman A., A. Meltzer, 1991, A political theory of progressive income taxation, in Political Economy, by A. Meltzer, A. Cukierman and S.F. Richard.(Eds.). Oxford University Press. New York.

\footnotetext{
${ }^{6}$ See De Donder et al (2000) for a presentation and a set-theoretical comparison of these different solution concepts
} 
[2] De Donder P. and J. Hindriks, 2000, The politics of progressive income taxation with incentive effects, mimeo IDEI.

[3] De Donder P., M. Le Breton and M. Truchon, 2000, Choosing in a weighted tournament, Mathematical Social science, forthcoming.

[4] Snyder, J. and G. Kramer, 1988, Fairness, self-interest, and the politics of the progressive income tax, Journal of Public economics, 36, 197-230.

[5] Marhuenda, F., I. Ortuno-Ortin, 1995, Popular support for progressive taxation, Economics Letters,48, 319-24.

[6] Mitra, T., E. Ok, L. Kockesen, 1998, Popular support for progressive taxation and the relative income hypothesis, Economics Letters, 58, 69-76.

[7] Myles, G., 2000, On the optimal marginal rate of income tax, Economics Letters,113-119.

[8] Roemer, J., 1999, The democratic political economy of progressive income taxation, Econometrica, 67, 1-19.

[9] Young, H.P.,1990, Progressive taxation and equal sacrifice, American Economic Review, 80, 253-66. 